\title{
Valve-in-valve procedure after CoreValve pop-out
}

\author{
Aleksandra Gasecka, Aleksander Kotulecki, Kacper Gałuszka, Zenon Huczek, Piotr Ścisło, Bartosz Rymuza, \\ Janusz Kochman
}

$1^{\text {st }}$ Chair and Department of Cardiology, Medical University of Warsaw, Warsaw, Poland

Adv Interv Cardiol 2021; 17, 3 (65): 324-326

DOI: https://doi.org/10.5114/aic.2021.109157

Aortic stenosis (AS) is one of the most common valvular pathologies in the developed countries. Transcatheter aortic valve implantation (TAVI) has become an accepted treatment for severe AS in high and intermediate risk patients, as an alternative to surgical aortic valve replacement (SAVR) [1]. Although TAVI is a less invasive procedure than SAVR, it carries a risk of complications including valve misplacement or embolization [2].

A 72-year-old man with a complex aortic valve defect including low-gradient, low-flow severe AS (max/mean gradient $34 / 20 \mathrm{~mm} \mathrm{Hg}, \mathrm{V} \max 2.9 \mathrm{~m} / \mathrm{s}$, AVAi $0.44 \mathrm{~cm}^{2} / \mathrm{m}^{2}$ ) and aortic regurgitation, with a history of myocardial infarction treated with coronary artery bypass grafting 15 years earlier, chronic heart failure with a mid-range ejection fraction, permanent atrial fibrillation, hypertension and diabetes mellitus, was admitted for interventional treatment of AS. Regarding the substantial perioperative death risk according to the EuroSCORE II (4.22\%), and the previous open-heart surgery, he was qualified for TAVI by the Heart Team. Computed tomography angiography was performed to assess the vascular access routes and valve anatomy, showing moderate valve calcifications (Figure $1 \mathrm{~A}$ ). The minimal and maximal diameters of the aortic annulus were $21.7 \mathrm{~mm}$ and $27.0 \mathrm{~mm}$, respectively, and the perimeter was $77 \mathrm{~mm}$ (Figure $1 \mathrm{~B}$ ).

The patient underwent TAVI via a transfemoral approach under local anesthesia. The self-expandable CoreValve $29 \mathrm{~mm}$ was positioned properly under fluoroscopy and the deployment started as usually 3-4 mm under the aortic annulus (Figure $1 \mathrm{C}$ ). Immediately after final deployment, the valve dived into the left ventricle outflow tract, resulting in severe paravalvular regurgitation (Figure $1 \mathrm{D}$ ). The decision to deploy the second CoreValve $29 \mathrm{~mm}$ was quickly made (Figure $1 \mathrm{E}$ ). Both valves were stable and the second valve was functional, with no signs of coronary vessel obstruction. Transesophageal echocar- diography after the second valve implantation showed mild paravalvular regurgitation (Figure $1 \mathrm{~F}$ ) and mild mitral stenosis (mitral valve area $2.2 \mathrm{~cm}^{2}$, mean gradient $2 \mathrm{~mm} \mathrm{Hg}$ ). The procedure was deemed successful and the further clinical course was uneventful. The patient was discharged from the hospital 7 days later.

Transcatheter heart valve embolization is a rare and serious complication of TAVI with a reported incidence of $0.3 \%$, associated with higher mortality and stroke rates [3]. The migration to the left ventricle correlates with worse outcomes (43\% mortality). While being a non-invasive procedure, TAVI offers a limited number of solutions regarding embolized valves.

Depending on the type of valve, there are several procedures that can either reposition, stabilize or remove a misplaced valve. In the case of embolization of balloon-expandable valves, a partially inflated valvuloplasty balloon can be used to grab the valve and drag it back into place [4]. In self-expandable valves, an attempt with a balloon or with lasso device can also be made to move a valve. An additional procedure to increase the safety of CoreValve TAVI is to implant the valve during rapid pacing (100-120 bpm). However, if misplaced, CoreValve and other self-expandable valves are hard to reposition, and implantation of a second valve is often required [4]. The latter approach increases the risk of coronary occlusion and poses a challenge if percutaneous coronary intervention is needed. However, it allows one to avoid surgical intervention.

Altogether, TAVI is a viable and less invasive approach to treat severe AS. In the case of valve embolization or misplacement, it is feasible to successfully implant a second valve.

\section{Conflict of interest}

The authors declare no conflict of interest.

\section{Corresponding author:}

Aleksandra Gasecka MD, PhD, 15t Chair and Department of Cardiology, Medical University of Warsaw, Warsaw, Poland, phone: +48 225991951 , e-mail: aleksandra.gasecka@wum.edu.pl

Received: 25.04.2021, accepted: 9.07.2021. 

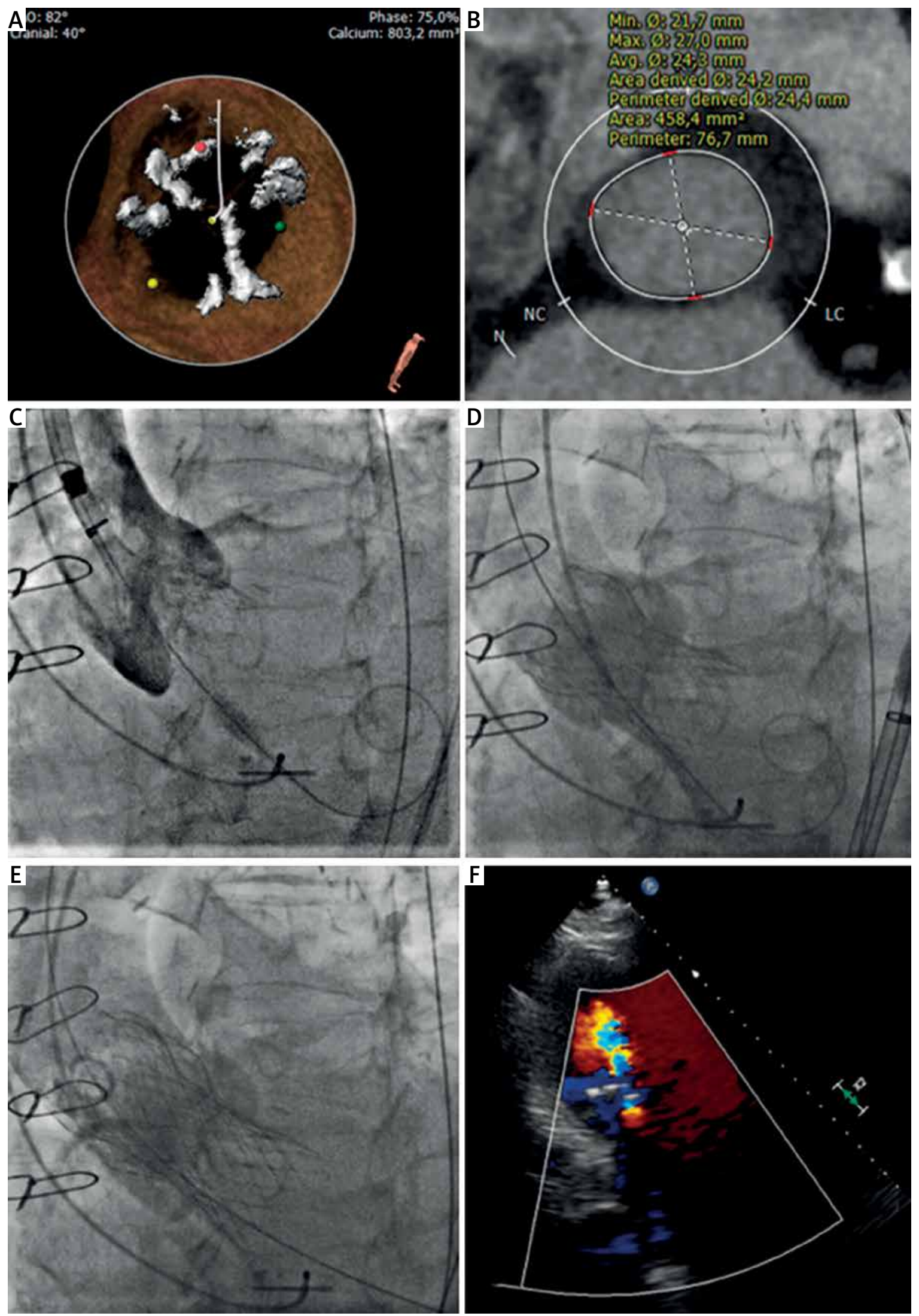

Figure 1. A - Angiography showing the deployment of the first CoreValve $(29 \mathrm{~mm})$. B - CoreValve $(29 \mathrm{~mm})$ pop-out into the left ventricle. C - Deployment of the second CoreValve $(29 \mathrm{~mm})$. D - Transesophageal echocardiography after the second valve implantation showing mild paravalvular regurgitation $(\mathrm{E}, \mathrm{F})$ 


\section{References}

1. Thonghong T, De Backer O, Søndergaard L. Comprehensive update on the new indications for transcatheter aortic valve replacement in the latest 2017 European guidelines for the management of valvular heart disease. Open Heart 2018; 5 : e000753.

2. Noorani A, Radia R, Bapat V. Challenges in valve-in-valve therapy. J Thorac Dis 2015; 7: 1501-8.

3. Ibebuogu UN, Giri S, Bolorunduro O, et al. Review of reported causes of device embolization following transcatheter aortic valve implantation. Am J Cardiol 2015; 115: 1767-72.

4. Kinthala S, Saththasivam P, Ankam A, Sattur S. Embolization of aortic valve leaflet during valve-in-valve transcatheter aortic valve implantation: a case report. Eur Heart J Case Rep 2020; 4: $1-5$. 